Acta Crystallographica Section E

Structure Reports

Online

ISSN 1600-5368

\section{(4-Hydroxy-3-methylphenyl)(phenyl)- methanone}

\section{S. Dileep, ${ }^{\text {a }}$ V. Lakshmi Ranganatha, ${ }^{\text {b }}$ N. K. Lokanath, ${ }^{a}$}

A. K. Shaukath ${ }^{\text {b }}$ and M. A. Sridhar ${ }^{\text {a* }}$

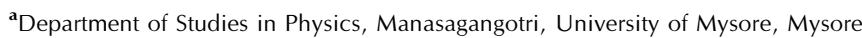
570 006, India, and 'Department of Chemistry, Yuvaraja's College, University of Mysore, Mysore 570 005, India

Correspondence e-mail: mas@physics.uni-mysore.ac.in

Received 7 September 2013; accepted 11 September 2013

Key indicators: single-crystal X-ray study; $T=293 \mathrm{~K}$; mean $\sigma(\mathrm{C}-\mathrm{C})=0.003 \AA$; $R$ factor $=0.046 ; w R$ factor $=0.129 ;$ data-to-parameter ratio $=12.4$.

In the title compound, $\mathrm{C}_{14} \mathrm{H}_{12} \mathrm{O}_{2}$, the benzene rings make a dihedral angle of $58.84(12)^{\circ}$. In the crystal, molecules are linked into chains along the $b$-axis direction by $\mathrm{O}-\mathrm{H} \cdots \mathrm{O}$ hydrogen bonds. These chains are further linked by $\mathrm{C}-\mathrm{H} \cdots \mathrm{O}$ hydrogen bonds, forming layers parallel to the $b c$ plane.

\section{Related literature}

For the biological activity of benzophenone derivatives, see: Khanum et al. (2004); Naveen et al. (2006); Selvi et al. (2003). For a related structure, see: Mahendra et al. (2005).<smiles>Cc1cc(C(=O)c2ccccc2)ccc1O</smiles>

\section{Experimental}

\section{Crystal data}

$\mathrm{C}_{14} \mathrm{H}_{12} \mathrm{O}_{2}$

$M_{r}=212.24$

Orthorhombic, $\mathrm{Pbca}$

$a=7.7043$ (4) $\AA$

$b=16.3770(8) \AA$

$c=17.7482(9) \AA$

$$
\begin{aligned}
& V=2239.4(2) \AA^{3} \\
& Z=8 \\
& \mathrm{Cu} K \alpha \text { radiation } \\
& \mu=0.67 \mathrm{~mm}^{-1} \\
& T=293 \mathrm{~K} \\
& 0.30 \times 0.25 \times 0.20 \mathrm{~mm}
\end{aligned}
$$

Data collection

Bruker X8 Proteum diffractometer

7657 measured reflections

1828 independent reflections

1518 reflections with $I>2 \sigma(I)$

$R_{\text {int }}=0.037$

Refinement

$R\left[F^{2}>2 \sigma\left(F^{2}\right)\right]=0.046$

$w R\left(F^{2}\right)=0.129$

$S=1.03$

1828 reflections

147 parameters

$\mathrm{H}$-atom parameters constrained

$\Delta \rho_{\max }=0.14 \mathrm{e} \AA^{-3}$

$\Delta \rho_{\min }=-0.15 \mathrm{e}^{-3}$

Table 1

Hydrogen-bond geometry $\left(\AA,^{\circ}\right)$.

\begin{tabular}{lllll}
\hline$D-\mathrm{H} \cdots A$ & $D-\mathrm{H}$ & $\mathrm{H} \cdots A$ & $D \cdots A$ & $D-\mathrm{H} \cdots A$ \\
\hline $\mathrm{O} 14-\mathrm{H} 14 \cdots \mathrm{O} 8^{\mathrm{i}}$ & 0.82 & 1.91 & $2.7106(19)$ & 166 \\
$\mathrm{C} 2-\mathrm{H} 2 \cdots \mathrm{O} 14^{\mathrm{ii}}$ & 0.93 & 2.57 & $3.448(3)$ & 158 \\
\hline
\end{tabular}

Symmetry codes: (i) $-x+\frac{1}{2}, y+\frac{1}{2}, z$; (ii) $x,-y+\frac{3}{2}, z-\frac{1}{2}$.

Data collection: APEX2 (Bruker, 2006); cell refinement: SAINT (Bruker, 2006); data reduction: $S A I N T$; program(s) used to solve structure: SHELXS97 (Sheldrick, 2008); program(s) used to refine structure: SHELXL97 (Sheldrick, 2008); molecular graphics: PLATON (Spek, 2009); software used to prepare material for publication: SHELXL97.

The authors would like to thank the University of Mysore for providing the diffractometer facility under IoE. VLR acknowledges the financial support provided by the Department of Science and Technology, New Delhi, under the INSPIRE-Fellowship scheme [IF110555]. SAK gratefully acknowledges the financial assistance provided by the UGC under the major research project scheme [F.39/737/2010 (SR)]. CSD would like to thank the University of Mysore for the award of an RFSMS fellowship under the head DV5/Physics/ 389/RFSMS/2009-2010/10.07.2012.

Supplementary data and figures for this paper are available from the IUCr electronic archives (Reference: IS5303).

\title{
References
}

Bruker (2006). APEX2 and SAINT. Bruker AXS Inc., Madison, Wisconsin, USA.

Khanum, S. A., Venu, T. D., Shashikanth, S. \& Firdouse, A. (2004). Bioorg. Med. Chem. Lett. 12, 2093-2095.

Mahendra, M., Khanum, S. A., Singh, A. K., Shashikanth, S., Doreswamy, B. H., Sridhar, M. A. \& Shashidhara Prasad, J. (2005). Acta Cryst. E61, o2990o2991.

Naveen, S., Khanum, S. A., Devaiah, V. T., Shashikanth, S., Anandalwar, S. M. \& Prasad, S. (2006). Anal. Sci. 22, 183-184.

Selvi, A. T., Joseph, G. S. \& Jayaprakasha, G. K. (2003). Food Microbiol. 20, 455-460.

Sheldrick, G. M. (2008). Acta Cryst. A64, 112-122.

Spek, A. L. (2009). Acta Cryst. D65, 148-155. 


\section{supporting information}

Acta Cryst. (2013). E69, o1550 [doi:10.1107/S160053681302521X]

\section{(4-Hydroxy-3-methylphenyl)(phenyl)methanone}

\section{S. Dileep, V. Lakshmi Ranganatha, N. K. Lokanath, A. K. Shaukath and M. A. Sridhar}

\section{S1. Comment}

Benzophenone and related compounds have a wide variety of biological activities such as anti-fungal and antiinflammatory (Khanum et al., 2004; Selvi et al., 2003). The presence of various substituents in the benzophenone nucleus is essential to determining the quantitative structure-activity relationships of these systems. The competence of benzophenones as chemotherapeutic agents, especially as inhibitors of HIV-1 reverse transcriptase RT, cancer and inflammation, is well established and their chemistry has been studied extensively. In addition, methyl-substituted benzophenones exhibit chemotherapeutical activity against fungi. Some studies were carried out to show that methylsubstituted benzophenones exhibit anti-fungal properties (Naveen et al., 2006). In view of its extensive background, the title compound was prepared and characterized by single-crystal X-ray diffraction.

In the molecular structure of the title compound (Fig. 1), bond lengths and angles do not show large deviations and are comparable with those reported for a similar structure (Mahendra et al., 2005). The dihedral angle between the two benzene rings $(\mathrm{C} 1-\mathrm{C} 6)$ and $(\mathrm{C} 9-\mathrm{C} 16)$ is $58.84(12)^{\circ}$. The crystal structure is stabilized by intermolecular $\mathrm{C}-\mathrm{H} \cdots \mathrm{O}$ and $\mathrm{O}$ $-\mathrm{H} \cdots \mathrm{O}$ hydrogen bonds (Table 1 \& Fig. 2).

\section{S2. Experimental}

The title compound was synthesized by Fries rearrangement. 3-Methylphenylbenzoate was treated with anhydrous aluminium chloride $(0.002 \mathrm{~mol})$ as a catalyst at $150-170{ }^{\circ} \mathrm{C}$ under without solvent condition for about $2-3 \mathrm{~h}$. Then the reaction mixture was cooled to room temperature and quenched with $6 \mathrm{~N} \mathrm{HCl}$ in the presence of ice water. The reaction mixture was stirred for about $2-4 \mathrm{~h}$, and the solid was filtered and recrystallized with acetonitrile to obtain the title compound.

\section{S3. Refinement}

All H-atoms were located in a difference map and then were positioned geometrically $(\mathrm{C}-\mathrm{H}=0.93-0.96 \AA$ and $\mathrm{O}-\mathrm{H}=$ $0.82 \AA)$. They were refined using a riding model with $U_{\text {iso }}(\mathrm{H})=1.2 U_{\text {eq }}(\mathrm{C})$ or $1.5 U_{\text {eq }}\left(\mathrm{O}, \mathrm{C}_{\text {methyl }}\right)$. 


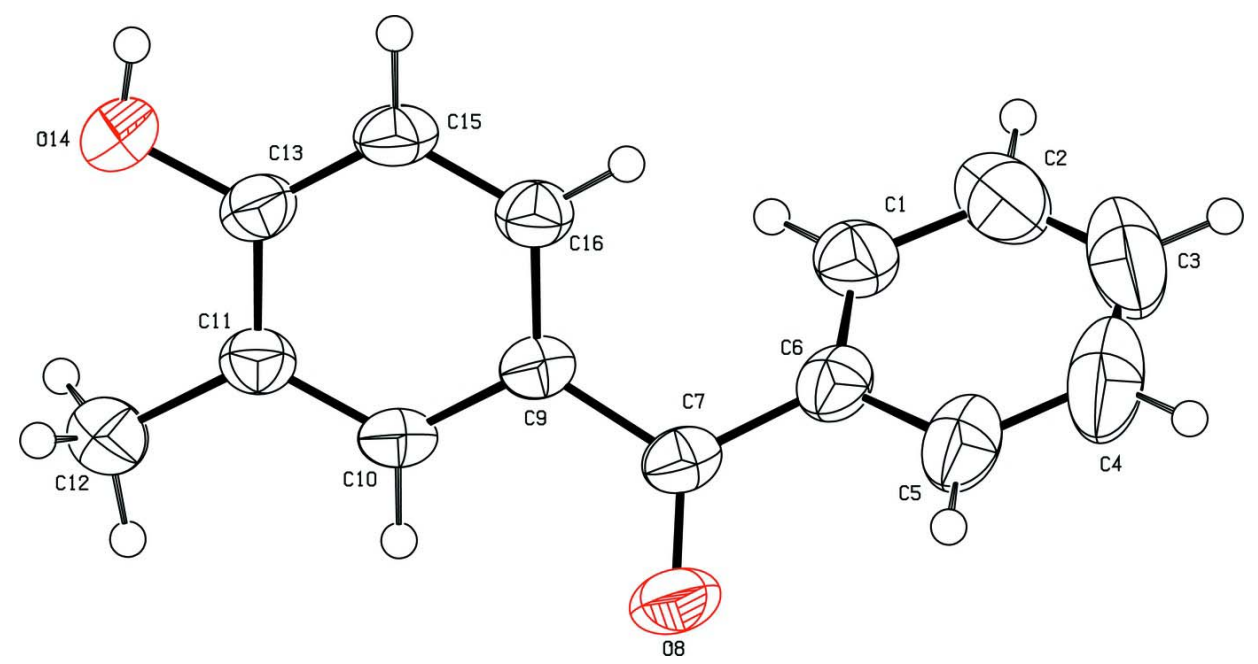

\section{Figure 1}

An ORTEP view of the molecule with the atom-labeling scheme. The thermal ellipsoids are drawn at the $50 \%$ probability level. $\mathrm{H}$ atoms are shown as small spheres of arbitrary radii.

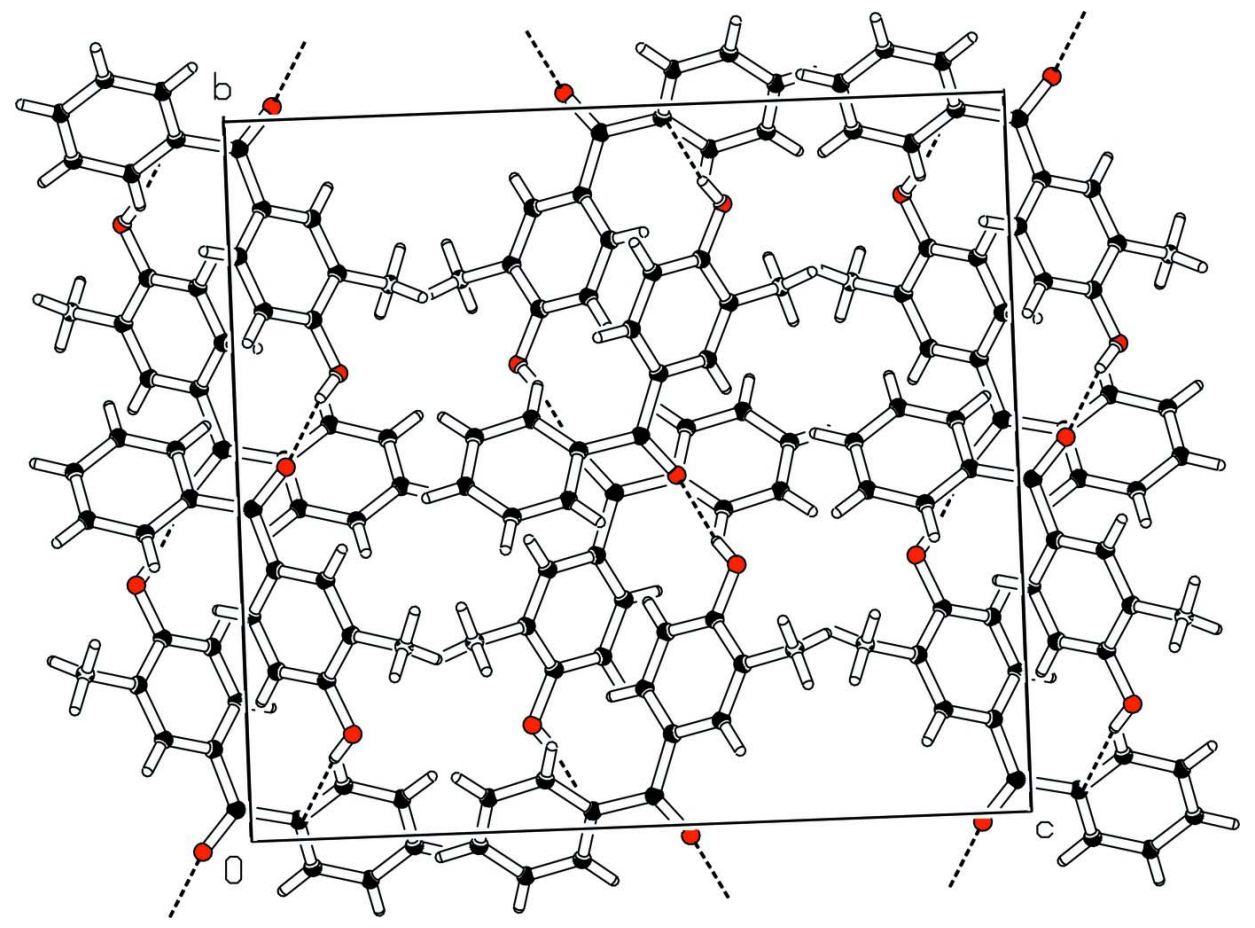

Figure 2

A molecular packing view of the title compound down the $a$-axis, showing intermolecular interactions (dashed lines).

\section{(4-Hydroxy-3-methylphenyl)(phenyl)methanone}

Crystal data

$\mathrm{C}_{14} \mathrm{H}_{12} \mathrm{O}_{2}$

$M_{r}=212.24$

Orthorhombic, $\mathrm{Pbca}$

Hall symbol: -P 2ac $2 \mathrm{ab}$

$$
\begin{aligned}
& a=7.7043(4) \AA \\
& b=16.3770(8) \AA \\
& c=17.7482(9) \AA \\
& V=2239.4(2) \AA^{3}
\end{aligned}
$$




\section{$Z=8$}

$F(000)=896$

$D_{\mathrm{x}}=1.259 \mathrm{Mg} \mathrm{m}^{-3}$

$\mathrm{Cu} K \alpha$ radiation, $\lambda=1.54178 \AA$

Cell parameters from 1828 reflections

\section{Data collection}

Bruker X8 Proteum

diffractometer

Radiation source: Bruker MicroStar microfocus rotating anode

Helios multilayer optics monochromator

Detector resolution: 10.7 pixels $\mathrm{mm}^{-1}$

$\varphi$ and $\omega$ scans

7657 measured reflections

\section{Refinement}

Refinement on $F^{2}$

Least-squares matrix: full

$R\left[F^{2}>2 \sigma\left(F^{2}\right)\right]=0.046$

$w R\left(F^{2}\right)=0.129$

$S=1.03$

1828 reflections

147 parameters

0 restraints

Primary atom site location: structure-invariant direct methods

Secondary atom site location: difference Fourier map $\theta=5.0-64.4^{\circ}$

$\mu=0.67 \mathrm{~mm}^{-1}$

$T=293 \mathrm{~K}$

Block, colorless

$0.30 \times 0.25 \times 0.20 \mathrm{~mm}$

1828 independent reflections

1518 reflections with $I>2 \sigma(I)$

$R_{\text {int }}=0.037$

$\theta_{\text {max }}=64.4^{\circ}, \theta_{\min }=5.0^{\circ}$

$h=-3 \rightarrow 8$

$k=-18 \rightarrow 18$

$l=-20 \rightarrow 20$
Hydrogen site location: inferred from neighbouring sites

$\mathrm{H}$-atom parameters constrained

$w=1 /\left[\sigma^{2}\left(F_{\mathrm{o}}^{2}\right)+(0.0617 P)^{2}+0.8504 P\right]$

where $P=\left(F_{\mathrm{o}}{ }^{2}+2 F_{\mathrm{c}}{ }^{2}\right) / 3$

$(\Delta / \sigma)_{\max }<0.001$

$\Delta \rho_{\max }=0.14 \mathrm{e} \AA^{-3}$

$\Delta \rho_{\min }=-0.15$ e $\AA^{-3}$

Extinction correction: SHELXL, $\mathrm{FC}^{*}=\mathrm{KFC}\left[1+0.001 \mathrm{XFC}^{2} \Lambda^{3} / \mathrm{SIN}(2 \Theta)\right]^{-1 / 4}$

Extinction coefficient: 0.0023 (3)

\section{Special details}

Geometry. Bond distances, angles etc. have been calculated using the rounded fractional coordinates. All su's are estimated from the variances of the (full) variance-covariance matrix. The cell e.s.d.'s are taken into account in the estimation of distances, angles and torsion angles

Refinement. Refinement on $F^{2}$ for ALL reflections except those flagged by the user for potential systematic errors. Weighted $R$-factors $w R$ and all goodnesses of fit $S$ are based on $F^{2}$, conventional $R$-factors $R$ are based on $F$, with $F$ set to zero for negative $F^{2}$. The observed criterion of $F^{2}>\sigma\left(F^{2}\right)$ is used only for calculating $-R$-factor-obs etc. and is not relevant to the choice of reflections for refinement. $R$-factors based on $F^{2}$ are statistically about twice as large as those based on $F$, and $R$-factors based on ALL data will be even larger.

Fractional atomic coordinates and isotropic or equivalent isotropic displacement parameters $\left(\AA^{2}\right)$

\begin{tabular}{lllll}
\hline & $x$ & $y$ & $z$ & $U_{\text {iso }} * U_{\text {eq }}$ \\
\hline O8 & $0.1870(2)$ & $0.48369(7)$ & $0.06235(8)$ & $0.0564(5)$ \\
O14 & $0.1579(2)$ & $0.85740(8)$ & $0.13455(8)$ & $0.0637(6)$ \\
C1 & $0.1850(3)$ & $0.56879(13)$ & $-0.12119(11)$ & $0.0553(7)$ \\
C2 & $0.2200(4)$ & $0.54665(17)$ & $-0.19461(13)$ & $0.0756(9)$ \\
C3 & $0.3252(4)$ & $0.4804(2)$ & $-0.20900(18)$ & $0.0944(11)$ \\
C4 & $0.3942(4)$ & $0.43527(19)$ & $-0.15054(18)$ & $0.0886(11)$ \\
C5 & $0.3563(3)$ & $0.45538(13)$ & $-0.07775(14)$ & $0.0623(8)$ \\
C6 & $0.2522(3)$ & $0.52263(11)$ & $-0.06186(11)$ & $0.0457(6)$ \\
C7 & $0.2081(2)$ & $0.54037(10)$ & $0.01775(10)$ & $0.0407(6)$ \\
C9 & $0.1909(2)$ & $0.62549(10)$ & $0.04420(10)$ & $0.0374(5)$
\end{tabular}




$\begin{array}{lllll}\text { C10 } & 0.0890(2) & 0.64224(10) & 0.10733(10) & 0.0385(5) \\ \text { C11 } & 0.0763(2) & 0.71935(10) & 0.13776(10) & 0.0412(6) \\ \text { C12 } & -0.0375(3) & 0.73782(12) & 0.20447(12) & 0.0624(8) \\ \text { C13 } & 0.1725(2) & 0.78203(10) & 0.10381(10) & 0.0426(6) \\ \text { C15 } & 0.2755(3) & 0.76672(11) & 0.04110(11) & 0.0456(6) \\ \text { C16 } & 0.2830(2) & 0.68939(11) & 0.01104(10) & 0.0431(6) \\ \text { H1 } & 0.11670 & 0.61430 & -0.11120 & 0.0660^{*} \\ \text { H2 } & 0.17280 & 0.57630 & -0.23430 & 0.0910^{*} \\ \text { H3 } & 0.35000 & 0.46600 & -0.25850 & 0.1130^{*} \\ \text { H4 } & 0.46650 & 0.39120 & -0.16080 & 0.1060^{*} \\ \text { H5 } & 0.40030 & 0.42390 & -0.03850 & 0.0750^{*} \\ \text { H10 } & 0.02750 & 0.59980 & 0.12970 & 0.0460^{*} \\ \text { H12A } & -0.09550 & 0.68880 & 0.22030 & 0.0940^{*} \\ \text { H12B } & -0.12210 & 0.77820 & 0.19070 & 0.0940^{*} \\ \text { H12C } & 0.03250 & 0.75820 & 0.24510 & 0.0940^{*} \\ \text { H14 } & 0.21640 & 0.88990 & 0.11020 & 0.0950^{*} \\ \text { H15 } & 0.33940 & 0.80880 & 0.01940 & 0.0550^{*} \\ \text { H16 } & 0.34990 & 0.67960 & -0.03160 & 0.0520^{*}\end{array}$

Atomic displacement parameters $\left(\AA^{2}\right)$

\begin{tabular}{lllllll}
\hline & $U^{11}$ & $U^{22}$ & $U^{33}$ & $U^{12}$ & $U^{13}$ & $U^{23}$ \\
\hline O8 & $0.0647(10)$ & $0.0356(7)$ & $0.0688(9)$ & $0.0039(6)$ & $0.0086(7)$ & $0.0147(6)$ \\
O14 & $0.0935(12)$ & $0.0353(7)$ & $0.0622(9)$ & $-0.0083(7)$ & $0.0170(8)$ & $-0.0035(6)$ \\
C1 & $0.0597(13)$ & $0.0525(11)$ & $0.0538(11)$ & $-0.0129(10)$ & $0.0040(10)$ & $0.0005(9)$ \\
C2 & $0.0883(19)$ & $0.0862(17)$ & $0.0524(12)$ & $-0.0340(15)$ & $0.0057(13)$ & $-0.0043(12)$ \\
C3 & $0.096(2)$ & $0.111(2)$ & $0.0763(18)$ & $-0.0373(19)$ & $0.0327(17)$ & $-0.0442(18)$ \\
C4 & $0.0747(18)$ & $0.0860(19)$ & $0.105(2)$ & $-0.0047(15)$ & $0.0238(17)$ & $-0.0464(18)$ \\
C5 & $0.0519(13)$ & $0.0530(12)$ & $0.0819(15)$ & $-0.0007(10)$ & $0.0089(12)$ & $-0.0169(11)$ \\
C6 & $0.0397(10)$ & $0.0400(10)$ & $0.0574(11)$ & $-0.0072(8)$ & $0.0047(9)$ & $-0.0047(8)$ \\
C7 & $0.0341(10)$ & $0.0342(9)$ & $0.0538(10)$ & $-0.0001(7)$ & $0.0016(8)$ & $0.0057(8)$ \\
C9 & $0.0352(9)$ & $0.0332(9)$ & $0.0438(9)$ & $-0.0001(7)$ & $-0.0005(7)$ & $0.0061(7)$ \\
C10 & $0.0381(10)$ & $0.0347(9)$ & $0.0428(9)$ & $-0.0014(7)$ & $0.0007(8)$ & $0.0109(7)$ \\
C11 & $0.0440(11)$ & $0.0389(9)$ & $0.0407(9)$ & $0.0017(7)$ & $0.0011(8)$ & $0.0068(7)$ \\
C12 & $0.0773(16)$ & $0.0518(12)$ & $0.0582(12)$ & $0.0033(11)$ & $0.0216(11)$ & $0.0039(10)$ \\
C13 & $0.0496(11)$ & $0.0332(9)$ & $0.0451(10)$ & $0.0001(8)$ & $-0.0030(8)$ & $0.0041(7)$ \\
C15 & $0.0468(11)$ & $0.0375(10)$ & $0.0526(10)$ & $-0.0088(8)$ & $0.0057(9)$ & $0.0097(8)$ \\
C16 & $0.0419(11)$ & $0.0398(9)$ & $0.0476(10)$ & $-0.0025(8)$ & $0.0061(8)$ & $0.0054(8)$ \\
& & & & & &
\end{tabular}

Geometric parameters $\left(\AA,{ }^{\circ}\right)$

\begin{tabular}{llll}
\hline $\mathrm{O} 8-\mathrm{C} 7$ & $1.231(2)$ & $\mathrm{C} 11-\mathrm{C} 13$ & $1.402(2)$ \\
$\mathrm{O} 14-\mathrm{C} 13$ & $1.354(2)$ & $\mathrm{C} 13-\mathrm{C} 15$ & $1.390(3)$ \\
$\mathrm{O} 14-\mathrm{H} 14$ & 0.8200 & $\mathrm{C} 15-\mathrm{C} 16$ & $1.375(3)$ \\
$\mathrm{C} 1-\mathrm{C} 6$ & $1.396(3)$ & $\mathrm{C} 1-\mathrm{H} 1$ & 0.9300 \\
$\mathrm{C} 1-\mathrm{C} 2$ & $1.379(3)$ & $\mathrm{C} 2-\mathrm{H} 2$ & 0.9300 \\
$\mathrm{C} 2-\mathrm{C} 3$ & $1.378(4)$ & $\mathrm{C} 3-\mathrm{H} 3$ & 0.9300 \\
$\mathrm{C} 3-\mathrm{C} 4$ & $1.380(4)$ & $\mathrm{C} 4-\mathrm{H} 4$ & 0.9300
\end{tabular}




\begin{tabular}{|c|c|c|c|}
\hline $\mathrm{C} 4-\mathrm{C} 5$ & $1.365(4)$ & $\mathrm{C} 5-\mathrm{H} 5$ & 0.9300 \\
\hline $\mathrm{C} 5-\mathrm{C} 6$ & $1.391(3)$ & $\mathrm{C} 10-\mathrm{H} 10$ & 0.9300 \\
\hline $\mathrm{C} 6-\mathrm{C} 7$ & $1.482(3)$ & $\mathrm{C} 12-\mathrm{H} 12 \mathrm{~A}$ & 0.9600 \\
\hline $\mathrm{C} 7-\mathrm{C} 9$ & $1.477(2)$ & $\mathrm{C} 12-\mathrm{H} 12 \mathrm{~B}$ & 0.9600 \\
\hline $\mathrm{C} 9-\mathrm{C} 16$ & $1.395(2)$ & $\mathrm{C} 12-\mathrm{H} 12 \mathrm{C}$ & 0.9600 \\
\hline $\mathrm{C} 9-\mathrm{C} 10$ & $1.395(2)$ & $\mathrm{C} 15-\mathrm{H} 15$ & 0.9300 \\
\hline $\mathrm{C} 10-\mathrm{C} 11$ & $1.377(2)$ & $\mathrm{C} 16-\mathrm{H} 16$ & 0.9300 \\
\hline $\mathrm{C} 11-\mathrm{C} 12$ & $1.504(3)$ & & \\
\hline $\mathrm{O} 8 \cdots \mathrm{C}^{\mathrm{i}}$ & $3.385(3)$ & $\mathrm{C} 13 \cdots \mathrm{H} 16^{\mathrm{vii}}$ & 2.8700 \\
\hline $\mathrm{O} 8 \cdots \mathrm{C} 7^{\mathrm{i}}$ & $3.383(2)$ & $\mathrm{C} 16 \cdots \mathrm{H} 1$ & 2.8000 \\
\hline $\mathrm{O} 8 \cdots \mathrm{C} 1^{\mathrm{i}}$ & $3.169(3)$ & $\mathrm{H} 1 \cdots \mathrm{C} 9$ & 2.8200 \\
\hline $\mathrm{O} 8 \cdots \mathrm{O} 14^{\mathrm{ii}}$ & $2.7106(19)$ & $\mathrm{H} 1 \cdots \mathrm{C} 16$ & 2.8000 \\
\hline $\mathrm{O} 14 \cdots \mathrm{O}^{\mathrm{iii}}$ & 2.7106 (19) & $\mathrm{H} 1 \cdots \mathrm{H} 16$ & 2.5200 \\
\hline $\mathrm{O} 8 \cdots \mathrm{H} 10$ & 2.5600 & $\mathrm{H} 2 \cdots \mathrm{O} 14^{\text {viii }}$ & 2.5700 \\
\hline $\mathrm{O} 8 \cdots \mathrm{H} 5$ & 2.6200 & $\mathrm{H} 3 \cdots \mathrm{C} 10^{\text {ix }}$ & 3.0100 \\
\hline $\mathrm{O} 8 \cdots \mathrm{H} 14^{\mathrm{ii}}$ & 1.9100 & $\mathrm{H} 3 \cdots \mathrm{H} 10^{\text {ix }}$ & 2.4500 \\
\hline $\mathrm{O} 14 \cdots \mathrm{H} 12 \mathrm{~B}$ & 2.7100 & $\mathrm{H} 5 \cdots \mathrm{O} 8$ & 2.6200 \\
\hline $\mathrm{O} 14 \cdots \mathrm{H} 12 \mathrm{C}$ & 2.7200 & $\mathrm{H} 5 \cdots \mathrm{C}^{\mathrm{v}}$ & 3.1000 \\
\hline $\mathrm{O} 14 \cdots \mathrm{H} 2^{\mathrm{iv}}$ & 2.5700 & $\mathrm{H} 10 \cdots \mathrm{O} 8$ & 2.5600 \\
\hline $\mathrm{C} 1 \cdots \mathrm{C} 16$ & $3.159(3)$ & $\mathrm{H} 10 \cdots \mathrm{H} 12 \mathrm{~A}$ & 2.3700 \\
\hline $\mathrm{C} 1 \cdots \mathrm{O}^{\mathrm{i}}$ & $3.169(3)$ & $\mathrm{H} 10 \cdots 3^{\mathrm{vi}}$ & 2.4500 \\
\hline $\mathrm{C} 5 \cdots \mathrm{C} 7^{\mathrm{v}}$ & $3.522(3)$ & $\mathrm{H} 12 \mathrm{~A} \cdots \mathrm{H} 10$ & 2.3700 \\
\hline $\mathrm{C} 6 \cdots \mathrm{O}^{\mathrm{i}}$ & $3.385(3)$ & $\mathrm{H} 12 \mathrm{~B} \cdots \mathrm{O} 14$ & 2.7100 \\
\hline $\mathrm{C} 7 \cdots \mathrm{C} 7^{\mathrm{i}}$ & $3.525(2)$ & $\mathrm{H} 12 \mathrm{C} \cdots \mathrm{O} 14$ & 2.7200 \\
\hline $\mathrm{C} 7 \cdots \mathrm{C}^{\mathrm{v}}$ & $3.522(3)$ & 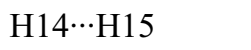 & 2.2900 \\
\hline $\mathrm{C} 7 \cdots \mathrm{O}^{\mathrm{i}}$ & $3.383(2)$ & $\mathrm{H} 14 \cdots \mathrm{O} 8^{\mathrm{iii}}$ & 1.9100 \\
\hline $\mathrm{C} 16 \cdots \mathrm{C} 1$ & $3.159(3)$ & $\mathrm{H} 14 \cdots \mathrm{C} 7^{\mathrm{iii}}$ & 3.0200 \\
\hline $\mathrm{C} 1 \cdots \mathrm{H} 16$ & 2.7300 & $\mathrm{H} 15 \cdots \mathrm{H} 14$ & 2.2900 \\
\hline С6 $\cdots \mathrm{H} 16$ & 2.7300 & $\mathrm{H} 15 \cdots \mathrm{C} 10^{\mathrm{x}}$ & 3.0700 \\
\hline $\mathrm{C} 7 \cdots \mathrm{H} 14^{\mathrm{ii}}$ & 3.0200 & $\mathrm{H} 16 \cdots \mathrm{C} 1$ & 2.7300 \\
\hline $\mathrm{C} 7 \cdots \mathrm{H} 5^{v}$ & 3.1000 & $\mathrm{H} 16 \cdots \mathrm{C} 6$ & 2.7300 \\
\hline $\mathrm{C} 9 \cdots \mathrm{H} 1$ & 2.8200 & $\mathrm{H} 16 \cdots \mathrm{H} 1$ & 2.5200 \\
\hline $\mathrm{C} 10 \cdots \mathrm{H}^{\mathrm{vi}}$ & 3.0100 & $\mathrm{H} 16{ }^{\cdots} \mathrm{C} 11^{\mathrm{x}}$ & 3.0500 \\
\hline $\mathrm{C} 10 \cdots \mathrm{H} 15^{\mathrm{vii}}$ & 3.0700 & $\mathrm{H} 16 \cdots \mathrm{C}^{\prime} 3^{\mathrm{x}}$ & 2.8700 \\
\hline $\mathrm{C} 11 \cdots \mathrm{H} 16^{\mathrm{vii}}$ & 3.0500 & & \\
\hline $\mathrm{C} 13-\mathrm{O} 14-\mathrm{H} 14$ & 109.00 & $\mathrm{C} 9-\mathrm{C} 16-\mathrm{C} 15$ & $120.39(17)$ \\
\hline $\mathrm{C} 2-\mathrm{C} 1-\mathrm{C} 6$ & $119.9(2)$ & $\mathrm{C} 2-\mathrm{C} 1-\mathrm{H} 1$ & 120.00 \\
\hline $\mathrm{C} 1-\mathrm{C} 2-\mathrm{C} 3$ & $119.8(2)$ & $\mathrm{C} 6-\mathrm{C} 1-\mathrm{H} 1$ & 120.00 \\
\hline $\mathrm{C} 2-\mathrm{C} 3-\mathrm{C} 4$ & $120.6(3)$ & $\mathrm{C} 1-\mathrm{C} 2-\mathrm{H} 2$ & 120.00 \\
\hline $\mathrm{C} 3-\mathrm{C} 4-\mathrm{C} 5$ & $120.0(3)$ & $\mathrm{C} 3-\mathrm{C} 2-\mathrm{H} 2$ & 120.00 \\
\hline $\mathrm{C} 4-\mathrm{C} 5-\mathrm{C} 6$ & $120.4(2)$ & $\mathrm{C} 2-\mathrm{C} 3-\mathrm{H} 3$ & 120.00 \\
\hline $\mathrm{C} 1-\mathrm{C} 6-\mathrm{C} 7$ & $121.87(18)$ & $\mathrm{C} 4-\mathrm{C} 3-\mathrm{H} 3$ & 120.00 \\
\hline $\mathrm{C} 5-\mathrm{C} 6-\mathrm{C} 7$ & $118.73(18)$ & $\mathrm{C} 3-\mathrm{C} 4-\mathrm{H} 4$ & 120.00 \\
\hline $\mathrm{C} 1-\mathrm{C} 6-\mathrm{C} 5$ & $119.32(19)$ & $\mathrm{C} 5-\mathrm{C} 4-\mathrm{H} 4$ & 120.00 \\
\hline $\mathrm{O} 8-\mathrm{C} 7-\mathrm{C} 9$ & $119.71(16)$ & $\mathrm{C} 4-\mathrm{C} 5-\mathrm{H} 5$ & 120.00 \\
\hline $\mathrm{C} 6-\mathrm{C} 7-\mathrm{C} 9$ & $120.58(15)$ & $\mathrm{C} 6-\mathrm{C} 5-\mathrm{H} 5$ & 120.00 \\
\hline
\end{tabular}




\begin{tabular}{|c|c|c|c|}
\hline $\mathrm{O} 8-\mathrm{C} 7-\mathrm{C} 6$ & $119.71(15)$ & $\mathrm{C} 9-\mathrm{C} 10-\mathrm{H} 10$ & 119.00 \\
\hline $\mathrm{C} 7-\mathrm{C} 9-\mathrm{C} 10$ & $119.43(15)$ & $\mathrm{C} 11-\mathrm{C} 10-\mathrm{H} 10$ & 119.00 \\
\hline $\mathrm{C} 7-\mathrm{C} 9-\mathrm{C} 16$ & $121.90(15)$ & $\mathrm{C} 11-\mathrm{C} 12-\mathrm{H} 12 \mathrm{~A}$ & 109.00 \\
\hline $\mathrm{C} 10-\mathrm{C} 9-\mathrm{C} 16$ & $118.53(15)$ & $\mathrm{C} 11-\mathrm{C} 12-\mathrm{H} 12 \mathrm{~B}$ & 110.00 \\
\hline $\mathrm{C} 9-\mathrm{C} 10-\mathrm{C} 11$ & $122.36(15)$ & $\mathrm{C} 11-\mathrm{C} 12-\mathrm{H} 12 \mathrm{C}$ & 109.00 \\
\hline $\mathrm{C} 10-\mathrm{C} 11-\mathrm{C} 12$ & $122.32(15)$ & $\mathrm{H} 12 \mathrm{~A}-\mathrm{C} 12-\mathrm{H} 12 \mathrm{~B}$ & 110.00 \\
\hline $\mathrm{C} 12-\mathrm{C} 11-\mathrm{C} 13$ & $119.95(15)$ & $\mathrm{H} 12 \mathrm{~A}-\mathrm{C} 12-\mathrm{H} 12 \mathrm{C}$ & 109.00 \\
\hline $\mathrm{C} 10-\mathrm{C} 11-\mathrm{C} 13$ & $117.73(15)$ & $\mathrm{H} 12 \mathrm{~B}-\mathrm{C} 12-\mathrm{H} 12 \mathrm{C}$ & 109.00 \\
\hline $\mathrm{O} 14-\mathrm{C} 13-\mathrm{C} 11$ & $116.76(15)$ & $\mathrm{C} 13-\mathrm{C} 15-\mathrm{H} 15$ & 120.00 \\
\hline $\mathrm{O} 14-\mathrm{C} 13-\mathrm{C} 15$ & $122.31(15)$ & $\mathrm{C} 16-\mathrm{C} 15-\mathrm{H} 15$ & 120.00 \\
\hline $\mathrm{C} 11-\mathrm{C} 13-\mathrm{C} 15$ & $120.92(16)$ & $\mathrm{C} 9-\mathrm{C} 16-\mathrm{H} 16$ & 120.00 \\
\hline $\mathrm{C} 13-\mathrm{C} 15-\mathrm{C} 16$ & $120.05(17)$ & $\mathrm{C} 15-\mathrm{C} 16-\mathrm{H} 16$ & 120.00 \\
\hline $\mathrm{C} 6-\mathrm{C} 1-\mathrm{C} 2-\mathrm{C} 3$ & $-1.8(4)$ & $\mathrm{C} 6-\mathrm{C} 7-\mathrm{C} 9-\mathrm{C} 16$ & $-28.8(2)$ \\
\hline $\mathrm{C} 2-\mathrm{C} 1-\mathrm{C} 6-\mathrm{C} 5$ & $1.0(3)$ & $\mathrm{C} 7-\mathrm{C} 9-\mathrm{C} 10-\mathrm{C} 11$ & $175.79(15)$ \\
\hline $\mathrm{C} 2-\mathrm{C} 1-\mathrm{C} 6-\mathrm{C} 7$ & $-175.6(2)$ & $\mathrm{C} 16-\mathrm{C} 9-\mathrm{C} 10-\mathrm{C} 11$ & $0.1(3)$ \\
\hline $\mathrm{C} 1-\mathrm{C} 2-\mathrm{C} 3-\mathrm{C} 4$ & $0.8(5)$ & $\mathrm{C} 7-\mathrm{C} 9-\mathrm{C} 16-\mathrm{C} 15$ & $-174.39(17)$ \\
\hline $\mathrm{C} 2-\mathrm{C} 3-\mathrm{C} 4-\mathrm{C} 5$ & $1.0(5)$ & $\mathrm{C} 10-\mathrm{C} 9-\mathrm{C} 16-\mathrm{C} 15$ & $1.2(3)$ \\
\hline $\mathrm{C} 3-\mathrm{C} 4-\mathrm{C} 5-\mathrm{C} 6$ & $-1.8(4)$ & $\mathrm{C} 9-\mathrm{C} 10-\mathrm{C} 11-\mathrm{C} 12$ & $178.23(17)$ \\
\hline $\mathrm{C} 4-\mathrm{C} 5-\mathrm{C} 6-\mathrm{C} 1$ & $0.8(3)$ & $\mathrm{C} 9-\mathrm{C} 10-\mathrm{C} 11-\mathrm{C} 13$ & $-1.0(2)$ \\
\hline $\mathrm{C} 4-\mathrm{C} 5-\mathrm{C} 6-\mathrm{C} 7$ & $177.5(2)$ & $\mathrm{C} 10-\mathrm{C} 11-\mathrm{C} 13-\mathrm{O} 14$ & $179.90(15)$ \\
\hline $\mathrm{C} 1-\mathrm{C} 6-\mathrm{C} 7-\mathrm{O} 8$ & $142.2(2)$ & $\mathrm{C} 10-\mathrm{C} 11-\mathrm{C} 13-\mathrm{C} 15$ & $0.7(3)$ \\
\hline $\mathrm{C} 1-\mathrm{C} 6-\mathrm{C} 7-\mathrm{C} 9$ & $-38.4(3)$ & $\mathrm{C} 12-\mathrm{C} 11-\mathrm{C} 13-\mathrm{O} 14$ & $0.7(2)$ \\
\hline $\mathrm{C} 5-\mathrm{C} 6-\mathrm{C} 7-\mathrm{O} 8$ & $-34.4(3)$ & $\mathrm{C} 12-\mathrm{C} 11-\mathrm{C} 13-\mathrm{C} 15$ & $-178.57(18)$ \\
\hline $\mathrm{C} 5-\mathrm{C} 6-\mathrm{C} 7-\mathrm{C} 9$ & $145.05(18)$ & $\mathrm{O} 14-\mathrm{C} 13-\mathrm{C} 15-\mathrm{C} 16$ & $-178.60(17)$ \\
\hline $\mathrm{O} 8-\mathrm{C} 7-\mathrm{C} 9-\mathrm{C} 10$ & $-24.9(2)$ & $\mathrm{C} 11-\mathrm{C} 13-\mathrm{C} 15-\mathrm{C} 16$ & $0.6(3)$ \\
\hline $\mathrm{O} 8-\mathrm{C} 7-\mathrm{C} 9-\mathrm{C} 16$ & $150.64(17)$ & $\mathrm{C} 13-\mathrm{C} 15-\mathrm{C} 16-\mathrm{C} 9$ & $-1.5(3)$ \\
\hline $\mathrm{C} 6-\mathrm{C} 7-\mathrm{C} 9-\mathrm{C} 10$ & $155.66(17)$ & & \\
\hline
\end{tabular}

Symmetry codes: (i) $-x,-y+1,-z$; (ii) $-x+1 / 2, y-1 / 2, z$; (iii) $-x+1 / 2, y+1 / 2, z$; (iv) $x,-y+3 / 2, z+1 / 2$; (v) $-x+1,-y+1,-z$; (vi) $-x+1 / 2,-y+1, z+1 / 2$; (vii) $x-1 / 2,-y+3 / 2,-z$; (viii) $x,-y+3 / 2, z-1 / 2$; (ix) $-x+1 / 2,-y+1, z-1 / 2$; (x) $x+1 / 2,-y+3 / 2,-z$.

Hydrogen-bond geometry $\left(\AA,{ }^{\circ}\right)$

\begin{tabular}{lllll}
\hline$D-\mathrm{H} \cdots A$ & $D-\mathrm{H}$ & $\mathrm{H} \cdots A$ & $D \cdots A$ & $D-\mathrm{H} \cdots A$ \\
\hline $\mathrm{O} 14-\mathrm{H} 14 \cdots \mathrm{O} 8^{\text {iii }}$ & 0.82 & 1.91 & $2.7106(19)$ & 166 \\
$\mathrm{C} 2-\mathrm{H} 2 \cdots \mathrm{O} 14^{\text {viii }}$ & 0.93 & 2.57 & $3.448(3)$ & 158 \\
\hline
\end{tabular}

Symmetry codes: (iii) $-x+1 / 2, y+1 / 2, z$; (viii) $x,-y+3 / 2, z-1 / 2$. 